The following pages constitute the final, accepted and revised manuscript of the article:

Waninge, R., Kalda, E., Paulsson, M., Nylander, T., Bergenståhl, B., "Cryo-TEM of isolated milk fat globule membrane structures in cream", Physical Chemistry Chemical Physics, 2004, Vol: 6, Issue: 7, p 1518-23. Publisher: Royal Society of Chemistry

Use of alternative location to go to the published version of the article requires journal subscription.

Alternative location:

http://dx.doi.org/10.1039/b314613h 


\title{
Cryo-TEM of isolated milk fat globule membrane structures in creamy
}

\author{
Rianne Waninge, ${ }^{\text {a }}$ Evelin Kalda, ${ }^{a}$ Marie Paulsson, ${ }^{a}$ Tommy Nylander ${ }^{b}$ and Björn Bergenståhl ${ }^{a}$ \\ a Department of Food Technology, Engineering and Nutrition, Lund University, P.O. Box 124, \\ S-22100 Lund, Sweden. E-mail: Rianne.Waninge@livsteki.lth.se; Fax: b46 46222 9517; Tel: \\ p46 $462228311^{\text {b }}$ Department of Physical Chemistry 1, Lund University, P.O. Box 124, S- \\ 22100 Lund, Sweden
}

This study has focused on structures formed through the release of lipid membrane material from milk fat globules. The aim of the study was to describe vesicles in the cream plasma and processing-induced vesicular structures in cream and buttermilk. The dairy samples were divided into three fractions through centrifugation, using $\mathrm{D}_{2} \mathrm{O}$ for density control. These fractions were examined using cryogenic transmission electron microscopy (cryo-TEM). The cream layer contains submicron-sized emulsion droplets, which are spherical and partly covered with loosely associated protein aggregates. The structures of the coalescence-induced vesicles are mostly unilamellar but bilamellar and multilamellar vesicles are also present. Some spherical structures are observed but facetted particles dominate. In the buttermilk sample, spherical and slightly deformed vesicles are visible but no facetted structures are observed. The butter oil serum sample shows interesting membrane vesicle-fat globule aggregate. These types of structures were also visible in the skim milk sample. The results show that that vesicular material may be found in a range of dairy products. To our knowledge this is the first time that the existence of these types of structure has been unambiguously demonstrated in these systems.

Introduction Polar lipids in milk mainly originate from the milk fat globule membrane. The milk fat globule membrane (MFGM) sur- rounds the fat globules in unhomogenised milk. It acts as a natural emulsifying agent enabling the fat to remain dispersed in the aqueous phase of milk and preventing the coalescence of fat globules. ${ }^{1,2}$ The phospholipids in milk are concentrated in the MFGM itself or in other membranous particles that prob- ably originate from the MFGM. ${ }^{3}$

The structure of MFGM is still not known in detail, but it has been suggested that the fat globule surface is covered by a monolayer of polar lipids, which in turn is partly covered by a lipid bilayer. ${ }^{4}$ The inner layer is thought to form during the intracellular formation of the fat globule. The outer layer of the MFGM originates from the outer cell membrane (plasmalemma) and Golgi accumulating at the milk fat globule during the fat globule expulsion from the cells in the lactic gland. ${ }^{5}$ Different membrane proteins, such as MUC1, xanthine dehydrogenase/oxidase, butyrophilin and adipophilin, are parts of the MFGM. ${ }^{6}$

The main membrane lipids of the MFGM can be classified as zwitterionic polar lipids, i.e. phosphatidylcholine (PC), phosphatidylethanolamine (PE), sphingomyelin (SM), and as anionic lipids, i.e. phosphatidylinositol (PI) and phosphatidylserine (PS). The compositions of some dairy products are shown in Table 1. The compositions of the membrane lipids have both saturated and unsaturated fatty acids, while SM consists mainly of long saturated fatty acid chains. The MFGM lipids have a very low molecular solubility in both water and triglycerides ${ }^{7}$ but they are dispersible in water and in oil. They associate spontaneously and form various

y Presented at the 17th Conference of the European Colloid \& Interface Science Society, Firenze, Italy, September 21-26, 2003. liquid crystalline phases in contact with water. ${ }^{8}$ The typical membrane lipids form lamellar, cubic or reversed hexagonal liquid crystalline phases, depending on the lipid composition and temperature. ${ }^{9}$ The cubic phases are usually bicontinuous, where the lipids are organised in curved nonintersecting bilayers ${ }^{10}$ and are formed in conditions between a stable lamel- lar and a stable hexagonal phase. ${ }^{11}$ In the reversed hexagonal phase the lipids are organised in rod-shaped cylinders filled with water, where the lipid head groups are directed to the cen- tre of the cylinders. ${ }^{12} \mathrm{PE}$ forms mainly reversed hexagonal phases. In the lamellar phase the lipids are stacked in bilayers separated by water layers. ${ }^{12}$ Below the chain-melting tempera- ture the chains are frozen and the lamellar phase can be trans-formed into an a-gel. SM, with their long saturated chain lengths, readily form such a gel. ${ }^{13}$ In excess of water the mem- brane lipids forming a lamellar phase can be dispersed in vesicular form. Previously it has been shown that a mixture of membrane lipids resembling a model milk membrane lipid system mainly formed a lamellar phase. A reversed hexagona phase was pre- sent only at high PE content and a gel was only formed at high SM content. ${ }^{14}$

Table 1 Composition of the polar membrane lipids in milk and buttermilk and their main fatty acid residues (modified from Waninge et al. ${ }^{14}$ ).

Zwitterionic Anionic

\begin{tabular}{lllllll} 
Product & PC & PE & SM & PI & PS & Reference \\
\hline Buttermilk & 35 & 37 & 28 & 2 & 3 & 31 \\
Fresh milk & 25 & 34 & 24 & 6 & 3 & 3 \\
Main fatty acid residues 16:0 & $18: 1$ & $23: 0$ & & $18: 1$ & 32 \\
in membrane lipids 18:1 & $18: 2$ & $16: 0$ & & $18: 0$ & \\
from fresh milk & & $16: 0$ & & & & \\
\hline
\end{tabular}


Phase separation or domain formation in membrane lipid systems have been shown in several studies. Ekelund et al. ${ }^{15}$ showed that palmitic acid (C16) and lignoceric acid (C24) in a monolayer may display lateral phase separation when compressed below their melting point. An experimental and theoretical study by Garidel et al. ${ }^{16}$ showed that, in binary phospholipid-systems, small two-phase regions (gel $\mathrm{p}$ liquid crystalline phase) can be present if the high melting component is present at a sufficiently high concentration. Phase separation may also occur in the presence of cholesterol. ${ }^{17}$ It has been shown that lateral phase separation of cholesterol leads to deformed non-spherical vesicles.

Facetted vesicular structures have been observed by Mel 'nikova et al. ${ }^{19}$ in mixed systems of dioctadecyldimethylammonium bromide (DODAB) and dioleoyl phosphatidylethanolamine (DOPE) (4:1). Even in porcine SM from brain lipid extracts, facetted multilamellar structures have been observed below the chain melting point of the lipids. ${ }^{20}$ The presence of adsorbed macromolecules also may lead to facetted vesicular structures. ${ }^{21}$

The MFGM changes its composition and structure during processing. For instance, $20 \%$ of phospholipids from the fat globule membrane can disperse into the plasma during cooling. ${ }^{2}$ Furthermore, other processes such as heating, agitation with air inclusion and homogenisation change the outer layer of the MFGM and release membrane material to the milk plasma. Fig. 1 is a schematic diagram of the change in the MFGM. The partial triple layer disappears and a vesicle with membrane lipids and proteins, and a fat droplet, with a monolayer of membrane materials remains. In this study we focus on structures formed through release of membrane material from milk fat globules. The aim is to describe vesicles in the cream plasma and process-induced vesi- cular structures in cream and buttermilk. Our hypothesis is that the vesicle structure can be influenced by the membrane proteins and the various lipids present in the membrane.

\section{Materials and methods}

Sample preparation Commercial dairy cream (Skånemejerier, Malmö, Sweden) with $40 \%$ fat was obtained through a retailer. The milk was separated, the cream was kept for 30 min at $60 \mathrm{C}$, pasteurised at $95 \mathrm{C}$ and then cooled to $4 \mathrm{C}$. Commercial skim milk (Skå- nemejerier, Malmö, Sweden) with $0.1 \%$ fat (added as carrier of vitamins) was also obtained through a retailer. Samples of sweet buttermilk from the churning process, and buttermilk serum from butter oil production, were obtained directly from the process from Friesland Coberco Dairy Foods (Deventer, the Netherlands). The full experimental scheme is shown in Fig. 2. The cream was centrifuged at $12000 \mathrm{gn}_{\mathrm{n}}$ at $4 \mathrm{C}$ for 30 min to separate the cream from the plasma. After separation, the milk fat globule

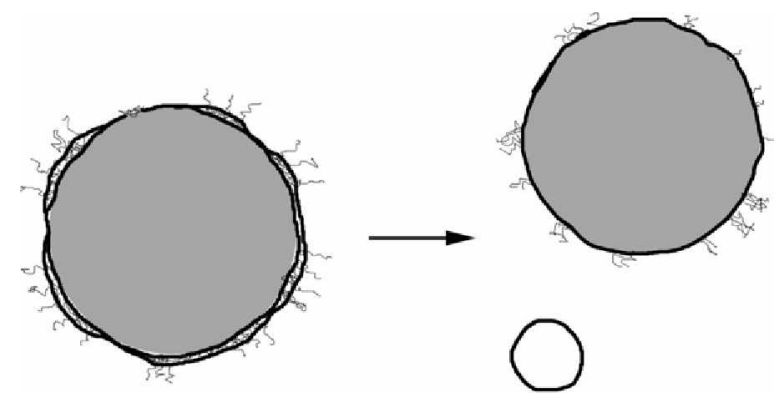

Fig. 1 A schematic diagram to show the change of the milk fat globule with a partly triple membrane to a vesicle and an emulsion droplet with a monolayer due to cooling, agitation, homogenisation etc.

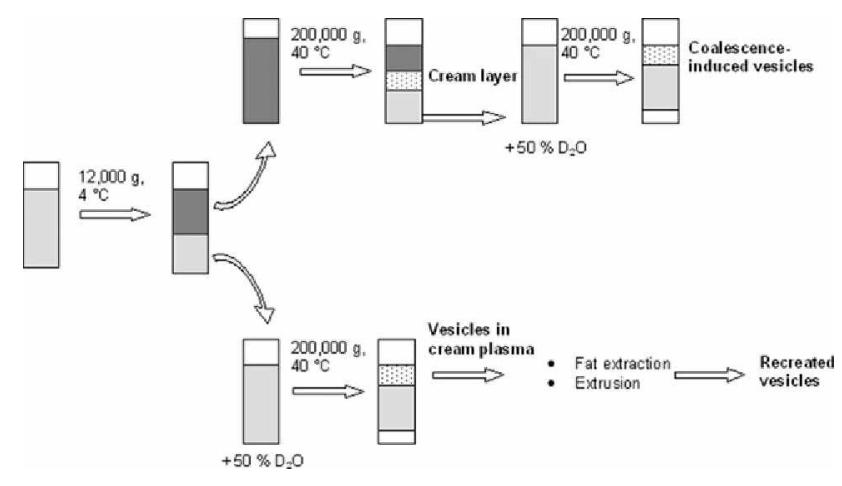

Fig. 2 Experimental set-up.

fraction was centrifuged for $1 \mathrm{~h}$ at $40 \mathrm{C}$, above the triglyceride melting temperature, to induce coalescence and release of the MFGM material.

A $20 \mathrm{mM}$ immidazole-HCl buffer with $50 \mathrm{mM} \mathrm{NaCl}, \mathrm{pH} 6.7$, with $50 \% \mathrm{D}_{2} \mathrm{O}$, was added to the subnatant (50:50). This gives a final density of about $1.04 \mathrm{~g} \mathrm{~cm}{ }^{3}$. The sample was centri- fuged at $40 \mathrm{C}, 200000 \mathrm{~g}$. Under these conditions the mem- brane lipids are expected to cream while the proteins still are expected to sediment. The upper layer was collected and used for further analyses. The same procedure was performed with the cream plasma. The upper layer was collected after centrifugation with heavy water, which contained vesicles. A fraction of this vesicular material was used to obtain a pure lipid fraction. A fat extrac- tion procedure was performed using the modified Röse-Got- tlieb method. ${ }^{22}$ Vesicles were recreated by extrusion through a $200 \mathrm{~nm}$ filter in a mini-extruder (Avanti Polar Lipids, Inc. Birmingham, $A L)$ at room temperature. The model recombined MFGM mixture was prepared using synthetic and natural lipids. The phospholipids, soy-phospha- tidylinositol (PI), dioleoylphosphatidylserine (DOPS), dioleoylphosphatidylethanolamine (DOPE), dioleoylphosphatidylcholine (DOPC) and milk sphingomyelin (SM) were purchased from Avanti Polar Lipids (Avanti Polar Lipids, Inc., Birmingham, $A L$ ) and were used without further purification

(99\% purity). Chloroform was used as a solvent for the phospholipids. The water used was passed through a MilliQ purification system (Millipore Corporation, Bedford, MA). Vesicles were prepared using a mini-extruder. Samples of the skim milk were analysed without further treatment.

Cryogenic transmission electron microscopy (Cryo-TEM) The samples for the cryo-TEM analyses were prepared in a controlled environment vitrification system (CEVS). The chamber temperature was $25 \mathrm{C}$ and the humidity was close to saturation to avoid evaporation in the sample during pre-paration. A small amount of the sample $(5 \mathrm{ml})$ was put on a lacy carbon film supported by a copper grid and gently blotted with filter paper to obtain a thin liquid film on the grid $(20-200 \mathrm{~nm})$. The grid was quenched in liquid ethane at $180 \mathrm{C}$ and transferred into liquid nitrogen ( 196 C). The technique is described in detail by Bellare et al. ${ }^{23}$ The vitrified specimens were stored under liquid nitrogen and transferred to a trans- mission electron microscope (Philips CM120 BioTWIN Cryo) equipped with a postcolumn energy filter (Gatan GIF 100), using an Oxford CT3500 cryoholder and its workstation. The acceleration voltage was $120 \mathrm{kV}$ and the working tempera- ture, 180 The images were recorded with a CCD camera

(Gatan 791) under low dose conditions, utilizing the zero loss peak (slit width of $9 \mathrm{eV}$ ). The defocus was approximately $1 \mathrm{~mm}$. The pictures are a two-dimensional picture of a three- dimensional structure. 
Results and discussion Fractions of vesicular and emulsion material in cream The vamples were treated with the objective of samples were treated with the objective of membrane material present in the cream plasma as well as the vesicular aggregates spontaneously formed during churning. The centrifugation in an aqueous phase with with a controlled density. By using heavy water we expected to be able to separate the vesicular material from proteins as well as from milk fat globules. Heavy water has a density of $1.11 \mathrm{~g} \mathrm{~cm} 3$, anhydrous lecithin $1.016 \mathrm{~g}$ $\mathrm{cm} 3$ and proteins about $1.34 \mathrm{~g}$ cm ${ }^{3}$. concentrated cream and a cream plasma phase through mild and cold centrifugation. The cream plasma phase, expecting to contain eventual vesicu- lar aggregates endogenous in the product, was treated
sepa- rately. The concentrated cream was centrifuged above the triglyceride melting temperature to induce coalescence and release of the milk fat globule membrane material associated with the fat globules. Antermediate white layer appeared, whiç, was collected and termed "cream layer". Most of the membrane lipids were expected to accumulate at the bottom of the tube during the centrifugation together with casein micelles. The membrane lipids were isolated by centrifu- gation with $\mathrm{D}_{2} \mathrm{O}$. The membrane lipid fraction was collected as a slightly turbid supernatant layer at the top and is termed

"coalescence induced vesicles". The membrane lipids were observed by cryo-TEM at room temperature. The cream plasma phase was also separated using a similar $\mathrm{D}_{2} \mathrm{O}$ centrifugation procedure. The vesicular containing frac- tion was collected as a slightly whitish layer on top of the subnatant and was termed "vesicles in the cream plasma". The division of the commercial cream into three fractions through centrifugation-the cream layer, coalescence induced vesicles and vesicles in the cream plasma- is also described in Fig. 2. These fractions were investigated by cryo-TEM.

Cream layer The cream layer was isolated as the intermediate fraction from cream after one hour of ultracentrifugation at 40 C. Most of the milk fat separated out as a separate liquid phase. From the intermediate cream fraction submicronsized emulsion droplets were present as shown by cryoTEM in Fig. 3. The micrograph shows submicron-sized emulsion

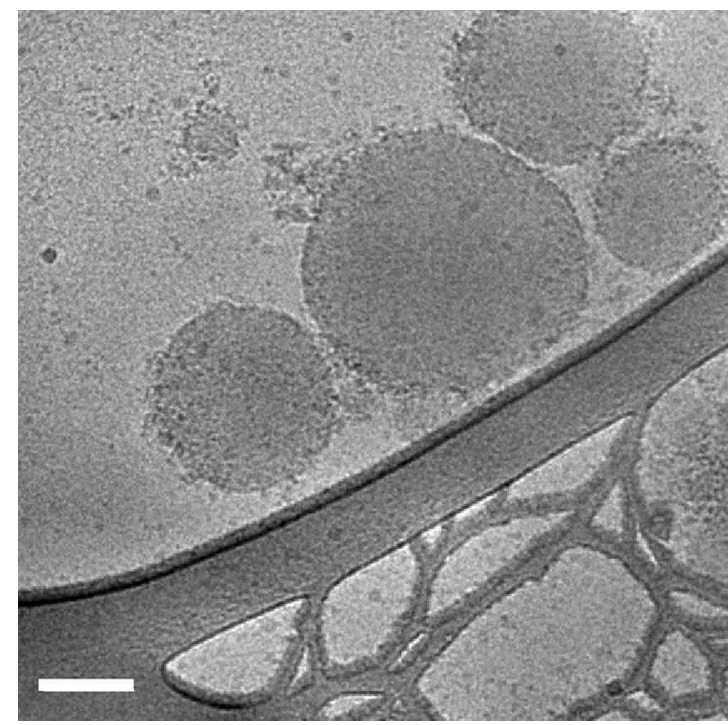

Fig. 3 Cryo-TEM image of submicron-sized emulsion droplets in the cream layer isolated from cream (see Fig. 2). Scale bar $100 \mathrm{~nm}$. droplets between 20 and $250 \mathrm{~nm}$. These droplets are spherical and seem to be partly covered with loosely associated protein aggregates. Based on the textural similarities to the appearance of casein micelles in cryo-TEM (casein micelles in skim milk, Fig. 4) we conclude that the loosely associated proteins are most prob- ably casein or casein micellar material adsorbed to the parti-cles. Loosely adsorbed proteins have also been observed by Buchheim and Dejmek. ${ }^{24}$ Other, more tightly adsorbed pro- teins may also be present but these protein layers are possibly too thin or/and have a low contrast. The small particle size observed in this fraction is in contrast to the major fraction of the native milk fat globules in cream (around 3-4 mm

$\left.\left(d_{43}\right)\right)$. We also recall that standard dairy cream is not homogenized so these submicron emulsions droplets occur naturally in milk. Similar sized milk fat globules have also been observed in unhomogenised milk or cream using freeze fracture electron microscopy. ${ }^{24}$ Michalski et al. ${ }^{25}$ have studied size distributions of milk samples obtained after hand milking, machine milking and after early processing in the dairy plant (pumping and transporting) using light scattering techniques. They found that a very distinct fraction of very small fat globules, 30

$300 \mathrm{~nm}$, appears after transportation and pumping at the dairy plant without being present in the milk after milking. How- ever, Walstra ${ }^{26}$ found that very small fat droplets are already present in the milk from the start. These results suggest that a fraction of the small fat globules is process induced and this may explain why casein can adhere to the submicron fat globules.

\section{Coalescence induced vesicles}

The cryo-TEM images of the coalescence-induced vesicles are shown in Figs. 5 and 6 . The structures are mostly unilamellar but bilamellar and multilamellar vesicles are also present. Some spherical structures are observed but facetted particles dominate. The repeat distance can be determined from the multilamellar vesicles. The Fourier transform showed that the repeat distance was $10 \mathrm{~nm}-4 \mathrm{~nm}$ from the bilayer itself and $6 \mathrm{~nm}$ from the water layer. Furthermore the vesicles appear to be non-aggregated and kept at a certain distance from each other.

The non-aggregated character of the vesicles suggests repulsive interactions operating between the aggregates. The

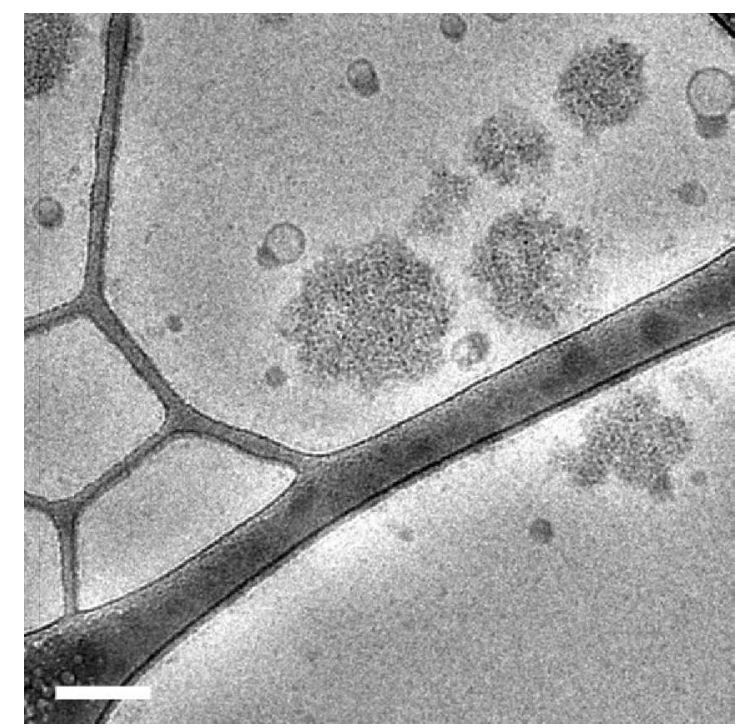

Fig. 4 Cryo-TEM image of vitaminised skim milk with casein micelles and vesicle-emulsion droplet aggregates (most likely formed by the carrier system for Vitamin A and D). Scale bar 100 $\mathrm{nm}$. 


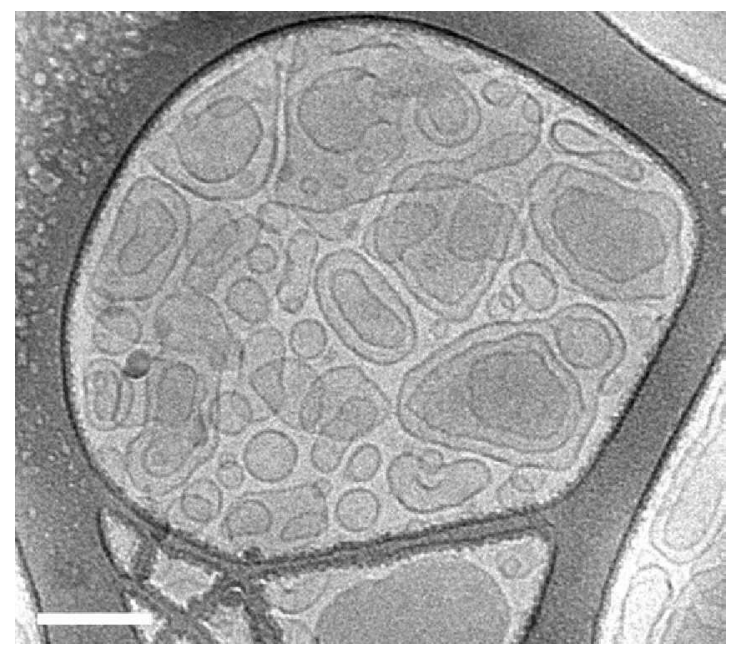

Fig. 5 Cryo-TEM image of coalescence-induced vesicles isolated from cream (see Fig. 2). Scale bar $100 \mathrm{~nm}$.

swelling to a repeat distance of $10 \mathrm{~nm}$ observed in the multilamellar vesicles supports this interpretation. In a previous study by Waninge et al. ${ }^{14}$ the repeat distance in a model milk membrane lipid system expanded from about $6 \mathrm{~nm}$ in absence of anionic lipids to $10 \mathrm{~nm}$ in presence of anionic lipids. This shows clearly that the presence of the anionic membrane lipids causes a swelling of the multilamellar assembly. The facetted character suggests an uneven lateral stress, surface tension, or bending modulus over the aggregate. This can be attributed to uneven composition, either of the lipids or of the proteins in or at the surface of the membrane.

Vesicles in the cream plasma and recreated vesicles The vesicles in the cream plasma were collected from the sub- natant of the centrifuged cream through centrifugation with heavy water. Fig. 7 shows a spherical vesicle with an internal deformed structure. It is clear that the non-aggregated (repulsive) character and the facetted surface typical for the coalescence-induced vesicles are also present in the vesicular structures in the cream plasma. The uneven composition in the MFGM material shown by the facetted structure may have different origin. A possible reason could be that parts of the

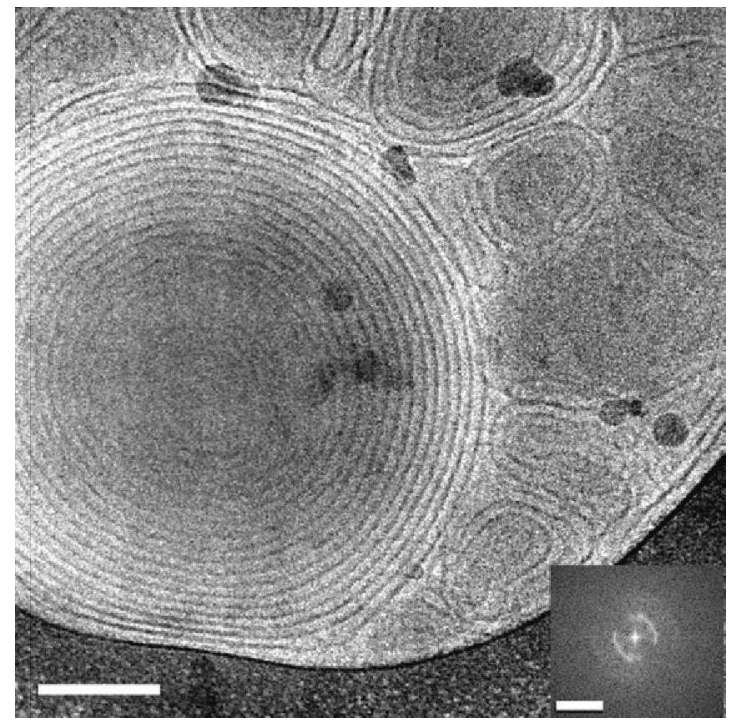

Fig. 6 Cryo-TEM image of coalescence-induced multilamellar vesicles (see Fig. 2) with its fast Fourier transform (FFT) pattern. Scale bar $100 \mathrm{~nm}$ (Scale bar FFT image $0.2 \mathrm{~nm}^{1}$ ).

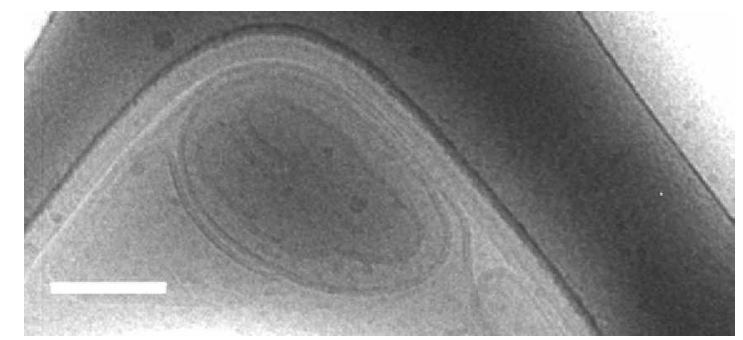

Fig. 7 Cryo-TEM image of a vesicle in the cream plasma (see Fig. 2). Scale bar $100 \mathrm{~nm}$.

chains of the lipid material are crystalline (in a-gel state) A second possibility is that cholesterol has phase-separated in the membrane. Other reasons could be that the protein frac- tion is unevenly distributed. To test the possibility of a lateral lipid phase separation, we investigated a recombined model MFGM lipid system (Fig. 8). ${ }^{27}$ In these systems we observed only spherical and deformed unilamellar, bilamellar and multi- lamellar structures without clear signs of a facetted character. This model MFGM sample contained saturated sphingomye- lin but no cholesterol. A second possible experiment was to prepare a microscopy sample at $40 \mathrm{C}$ (the attachment on the grid and blotting temperature). The result shows dominantly spherical morphologies (Fig. 9), indicating that the lateral het- erogeneities depend on the temperature. This may be caused either by melting partly crystalline domains or by dissolving a crystalline fragment in the fluid matrix. The third possibility is that the vesicular structures are influ- enced by the presence of membrane proteins. To test this we separated the lipids from the protein by fat extraction and, after evaporation, adding them to the buffer. The dispersion was then extruded through a $200 \mathrm{~nm}$ filter. The recreated vesi- cles are shown in Fig. 10. We observed unilamellar and sphe- rical morphologies as well as aggregate structures and tetrahedral vesicular nano-foam-like structures. The protein removal and the reorganisation of the membrane lipids have led to highly aggregated vesicular structures. There are signs of facetted structures in these images, although it is difficult to unambiguously exclude that these effects not are due to aggregation of the vesicles. The aggregating character of the redispersed extracted membrane lipids shows that the

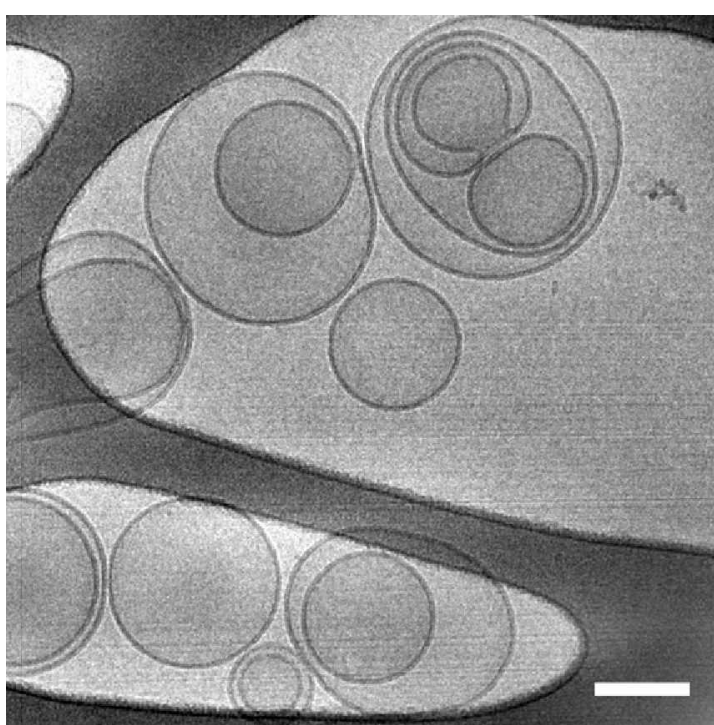

Fig. 8 Cryo-TEM image of model MFGM vesicles with composi- tion, 50 wt $\%$ DOPE, 43 wt $\%$ DOPC/SM (60/40), $7 \%$ DOPS/PI

$(50 / 50)$ ) in a $1 \mathrm{mM}$ phosphate buffer, $\mathrm{pH} 6.7$ with $70 \mathrm{mM} \mathrm{NaCl}$. Scale Bar $100 \mathrm{~nm}$ 


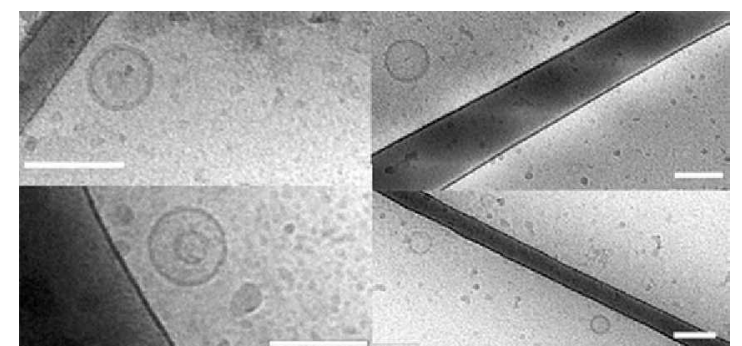

Fig. 9 Cryo-TEM image of coalescence-induced vesicles at $40 \mathrm{C}$ isolated from cream (see Fig. 2). Scale bar $100 \mathrm{~nm}$.

membranes with this composition are less able to form nonadhesive interactions. These results suggest that the membrane proteins have an important role in keeping the liposomal dispersion stable (Figs. 5-6). It is also likely that this stabilising effect from the membrane proteins also protects the fat globules of unhomogenised milk. The properties of the recreated vesicles are different from the ones produced from the model MFGM system (Fig. 8), which is also non-adhesive in its character. A possible explanation for this difference is the presence of cholesterol in the vesicles prepared from MFGM lipids isolated from cream.

Buttermilk and butter oil serum Buttermilk and butter oil serum samples obtained directly from the process in a dairy plant were treated in the same way as the subnatant of the cream. Figs. 11 and 12 show the respective cryo-TEM images of vesicles from buttermilk and butter oil serum, isolated accord- ing to Fig. 2. Note that the phospholipids content in these sam- ples was lower than in the cream samples so fewer objects are visible in the images. The buttermilk sample is dominated by spherical and slightly deformed vesicles (Fig. 11), but no

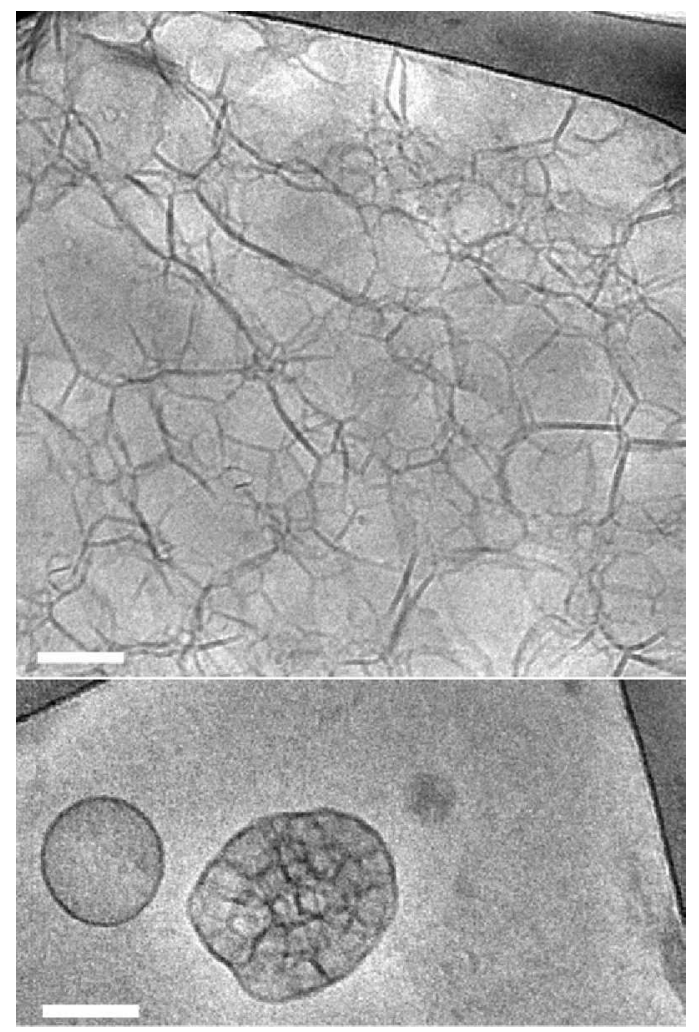

Fig. 10 Cryo-TEM images of recreated vesicles after fat extraction and extrusion (see Fig. 2). Scale bar $100 \mathrm{~nm}$
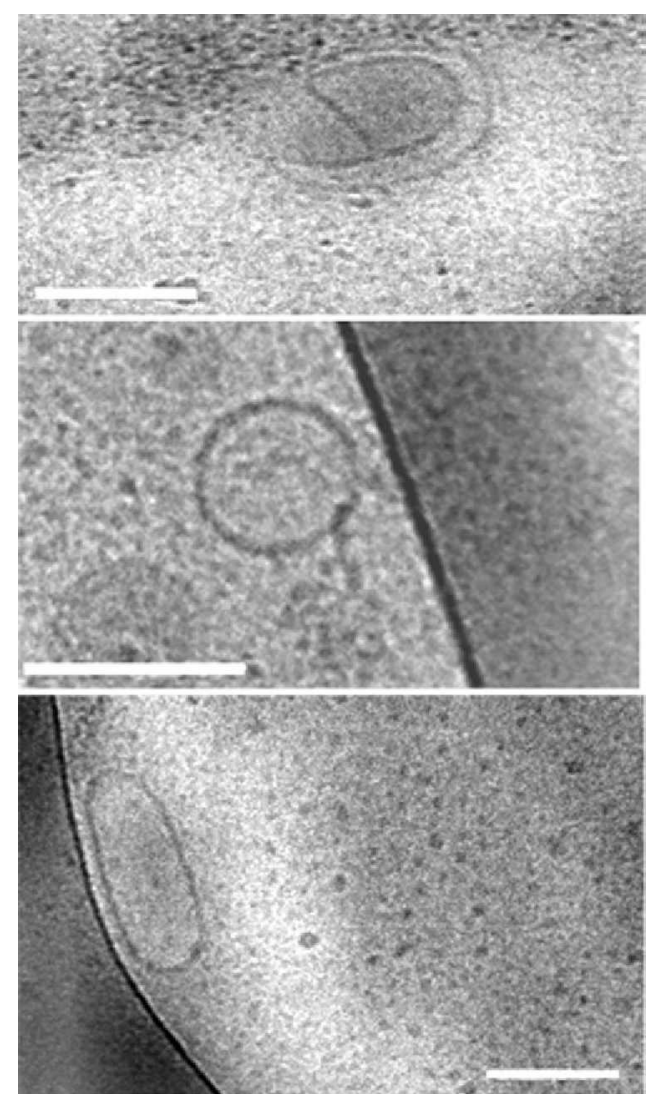

Fig. 11 Cryo-TEM image of vesicles isolated from buttermilk. Scale bar $100 \mathrm{~nm}$.

facetted structures are observed. The butter oil serum sample (Fig. 12) shows an interesting membrane vesicle-fat globule aggregate. These types of structures were also visible in the skim milk sample (Fig. 4). Teixeira et al. ${ }^{28}$ and Dahim et al. ${ }^{29}$ used cryo-TEM and showed vesicle-emulsion aggregates in cationic emulsions and in rapeseed oil and egg PC emulsions respectively. Such aggregates may have a technical significance. They will appear as one particle with a reduced density difference and thereby settle with a reduced rate compared to the original par- ticle. The interparticle interactions may also be reduced by the

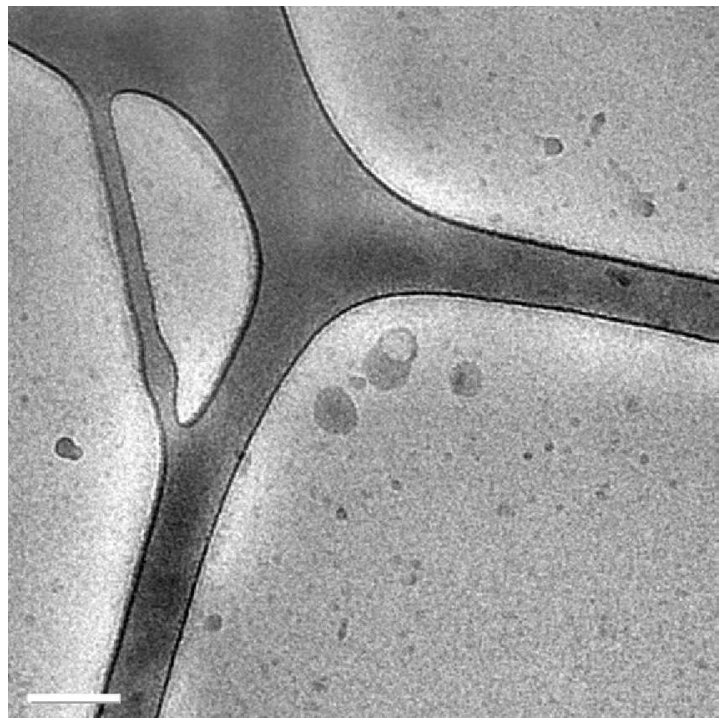

Fig. 12 Cryo-TEM image of a vesicle-emulsion droplet aggregate isolated from butter oil serum. Scale bar $100 \mathrm{~nm}$.

vesicular structures at the surface, preventing flocculation or loosing up flocculated structures making them more redisper- sible. A structural heterogeneity in terms of composition and densities in a commercial parental emulsion (a vegetable oil emulsified with lecithin) has been proposed by Venkatesh et al. ${ }^{30}$ who used field flow fractionation and observed vesicles, emulsion droplets and combined vesicle-emulsion droplets aggregates. The results show that vesicular material may be found in a range of dairy products or semi-manufactured products. To the best of our knowledge this is the first time that the existence of these types of structure have 
Conclusions From this study we can conclude the following: Vesicular membrane structures are present in several dairy products. They may be formed partly due to expulsion of the outer lipid bilayer or through coalescence of milk fat globules resulting in expulsion of membrane material. Both vesicles in the cream plasma and coalescence-induced vesicles appear in a non-aggregated state with more or less facetted structures.

The non-aggregating properties seem to be related to the anionic charge of phosphatidylinositol and phosphatidylserine and to the presence of membrane proteins in the native MFGM.

\section{References}

1 H. C. Deeth, Aust. J. Dairy Technol., 1997, 52, 44-46.

2 P. Walstra, T. J. Geurts, A. Noomen, A. Jellema and M. A. J. S. Van Boekel, Dairy Technology: Principles of Milk Properties and Processes, Dekker, New York, 1999.

3 W. W. Christie, R. C. Noble and G. Davies, J. Soc. Dairy

Technol., 1987, 40(1), 10-12.

4 T. E. Petersen, L. E. Rasmussen, E. S. Sorensen, J. T. Rasmussen, C. W. Heegaard, S. N. Fedosson, L. B. Johnsen, M. H. Andersen, L. B. Carsen and C. Benfeldt, in IDF Proceedings 1998, Mejeriforeningen, Århus, 1999, pp. 194-199.

5 S. Patton and T. W. Keenan, Biochim. Biophys. Acta, 1975, 415(3), 273-309.

6 I. H. Mather, J. Dairy Sci., 2000, 83, 203-247.

7 C. Tanford, The Hydrophobic Effect. Formation of Micelles \& Biological Membranes, John Wiley \& Sons, Inc., New York, 2nd edn., 1980.

8 K. Larsson, Lipids-Molecular Organization, Physical Functions and Technical Applications, Oily Press, Dundee, UK, 1994.

9 J. M. Seddon, Biochim. Biophys. Acta, 1990, 1031(1), 1-69.

10 G. Lindblom, K. Larsson, L. Johansson, K. Fontell and S. Forsen, J. Am. Chem. Soc., 1979, 101(19), 5465-5470.

11 K. Fontell, Mol. Cryst. Liq. Cryst., 1981, 63, 59-82.

12 V. Luzatti, X-Ray Diffraction Studies of Lipid-Water Systems, in Biological Membranes, ed. D. Chapman, Academic Press, London, 1968, p. 71-123.

13 M. Malmsten, B. Bergenståhl, L. Nyberg and G. Odham, J. Am. Oil Chem. Soc., 1994, 71(9), 1021-1026.

14 R. Waninge, T. Nylander, M. Paulsson and B. Bergenstahl, Chem. Phys. Lipids, 2003, 125(1), 59-68.

15 K. Ekelund, E. Sparr, J. Engblom, H. Wennerstrom and S. Engstrom, Langmuir, 1999, 15(20), 6946-6949.

16 P. Garidel, C. Johann and A. Blume, J. Liposome Res., 2000, 10(2-3), 131-158.

17 E. Sparr, K. Ekelund, J. Engblom, S. Engstrom and H. Wennerstrom, Langmuir, 1999, 15(20), 6950-6955.

18 K. Edwards, M. Johnsson, G. Karlsson and M. Silvander, Biophys. J., 1997, 73(1), 258-266.

19 Y. S. Mel'nikova, S. M. Mel'nikov and J. E. Lofroth, Biophys. Chem., 1999, 81(2), 125-141.

20 V. Alfredsson, K. Larsson, P. Lo Nostra, B. Ninham and T. Nylander, in 17th ECIS Conference, Book of Abstracts, European Colloid \& Interface Science Society, Fribourg, 2003, p. 469.

21 E. F. Marques, O. Regev, A. Khan, M. D. Miguel and B. Lindman, Macromolecules, 1999, 32(20), 6626-6637.

22 P. Walstra and H. Mulder, Neth. Milk Dairy J., 1962, 16(3), 172-184.

23 J. R. Bellare, H. T. Davis, L. E. Scriven and Y. Talmon, J. Electron Microsc. Tech., 1988, 10(1), 87-111.

24 W. Buchheim and P. Dejmek, in Food Emulsions, ed. S. E. Friberg, MarcelDekker, New York, 1990, p. 203-246.

25 M. C. Michalski, F. Michel and C. Geneste, Lait, 2002, 82(2), 193-208.

26 P. Walstra, Neth. Milk Dairy J., 1969, 23(2), 99-110.

27 R. Waninge, T. Nylander, M. Paulsson and B. Bergenstahl, Colloid Surf. B, 2003, 31(1-4), 257-264.

28 H. Teixeira, V. Rosilio, A. Laigle, J. Lepault, I. Erk, D. Scherman, S. Benita, P. Couvreur and C. Dubernet, Biophys. Chem., 2001, 92, 169-181.

29 M. Dahim, J. Gustafsson, F. Puisieux and M. Ollivon, Chem. Phys. Lipids, 1998, 97, 1-14.

30 S. Venkatesh, J. M. Li, K. D. Caldwell and B. D. Anderson, J. Pharm. Sci., 1998, 87(7), 859-866.

31 É. Dufour, M. Subirade, F. Loupil and A. Riaublanc, Lait, 1999, $79,217-228$.

32 W. R. Morrison, E. L. Jack and L. M. Smith, J. Am. Oil Chem. Soc., 1965, 42, 1142-1147. 